\title{
Utilidad de la angiografía retiniana por tomografía de coherencia óptica en la detección temprana de daño microvascular por diabetes
}

\section{Usefulness of optical coherence tomography angiography for detecting early diabetes-induced microvascular damage}

\author{
SHEYla L. Escobar-VILLAdo ${ }^{1}$ y Virgilio Lima-GÓMEZ ${ }^{*}$ \\ 'Escuela de Medicina, Universidad Autónoma de Ciudad Juárez, Ciudad Juárez, Chihuahua; ${ }^{2}$ Servicio de Oftalmología, Hospital Juárez \\ de México, Ciudad de México, México
}

\section{RESUMEN}

El daño microvascular causado por la diabetes en la retina es específico, pero sus manifestaciones clínicas se presentan tardíamente; existe daño capilar previo al desarrollo de retinopatía diabética, que no se puede descartar por la existencia de una imagen normal del fondo del ojo. La angiografía por tomografía de coherencia óptica es una herramienta de imagen que permite cuantificar la densidad de los capilares alrededor del área de mejor visión, y que ha identificado diferencias entre sujetos sin diabetes y pacientes diabéticos sin retinopatía. Se describen las métricas de la angiografía por tomografía de coherencia óptica más empleadas, y su aplicación para la evaluación de daño microvascular en estudios que analizan varios tejidos en pacientes con diabetes. Este estudio no invasivo permite determinar objetivamente los cambios en la microcirculación retiniana, y tanto detectar en forma temprana como descartar el daño en otros plexos capilares.

Palabras clave: Angiografía por tomografía de coherencia óptica. Densidad de vasos. Densidad de perfusión. Diabetes. Retina.

\begin{abstract}
Diabetes-induced microvascular retinal damage is specific, but its clinical features take time to appear; there is capillary damage before diabetic retinopathy starts, that cannot be ruled out with a normal ocular fundus image. Optical coherence tomography angiography is an imaging tool that allows to quantify the capillary density around the area of best visual function, and that has identified differences between non-diabetic people and diabetic patients without retinopathy. We describe the most used optical coherence tomography angiography metrics and their applications for evaluating microvascular damage in studies that analyze diverse tissues in patients with diabetes. This non-invasive study allows to objectively determine the changes in retinal microcirculation, and both detecting early and ruling out damage in other capillary plexuses.
\end{abstract}

Keywords: Diabetes. Optical coherence tomography angiography. Perfusion density. Retina. Vessel density.
Correspondencia:

*Virgilio Lima-Gómez

E-mail: forscher7@gmail.com
Fecha de recepción: 06-02-2021

Fecha de aceptación: 09-04-2021

DOI: 10.24875/RME.21000009
Disponible en internet: 22-02-2022

Rev Mex Endocrinol Metab Nutr. 2022;9:27-36

2462-4144 / @ 2021 Sociedad Mexicana de Nutrición y Endocrinología, AC. Publicado por Permanyer. Este es un artículo open access bajo la licencia CC BY-NC-ND (http://creativecommons.org/licenses/by-nc-nd/4.0/). 


\section{INTRODUCCIÓN}

En 2010 la Federación Internacional de Diabetes estimó que 285 millones de adultos de 20 a 79 años tenían diabetes mellitus (DM); para el 2040, se espera que este número casi se triplique y llegue a 642 millones $^{1}$. México se encuentra entre los países más afectados, con una prevalencia de diabetes tipo 2 de $9.4 \%$ en el 2016. Como en otros países de bajos ingresos, en México por cada persona con el diagnóstico de diabetes hay otra que tiene la enfermedad y lo desconoce; hay un amplio intervalo entre el inicio de la diabetes y su detección, que a su vez aumenta la frecuencia de complicaciones, como la retinopatía diabética $(\mathrm{RD})^{2}$.

La RD es una de las complicaciones microvasculares de la DM, actualmente es la principal causa mundial de ceguera y discapacidad visual en edad laboral ${ }^{3}$; la detección temprana y el tratamiento oportuno previenen hasta el $98 \%$ de la discapacidad visual relacionada con la DM. La RD se diagnostica mediante oftalmoscopia, con base en hallazgos como los microaneurismas, hemorragias retinianas, exudados y manchas algodonosas $^{4}$.

Aunque la RD es una enfermedad específica de la diabetes, aparece tardíamente, lo cual limita su uso como marcador temprano de microangiopatía; sin embargo, el daño microvascular retiniano inicia antes que las lesiones oftalmoscópicas, y actualmente puede cuantificarse mediante la angiografía por tomografía de coherencia óptica (angio-TCO), una tecnología que detecta el movimiento de los eritrocitos y permite delimitar la microvasculatura retiniana con una resolución equiparable a la de un corte histológi$\mathrm{CO}^{5,6}$.

El propósito de esta revisión es presentar los hallazgos vasculares encontrados mediante angio-TCO en pacientes diabéticos antes de que se desarrolle RD, para difundir su uso como un instrumento de detección temprana de daño microvascular en otros plexos capilares.
CAMBIOS MICROVASCULARES EN LA DIABETES ANTES DEL DESARROLLO DE RETINOPATÍA

Histológicamente el primer cambio causado por la DM en los capilares retinianos es el engrosamiento de la membrana basal endotelial ${ }^{7}$, a lo cual sigue la pérdida de pericitos. Los pericitos son células de sostén que participan en la regulación del flujo capilar, y que en condiciones normales rodean a las células endoteliales, con una relación 1:1; son sensibles a la hiperglucemia, que lleva a su muerte, lo que puede reducir su relación con respecto a las células endoteliales hasta 1:17. Este cambio en la pared lleva al colapso y al cierre capilar; junto a las zonas de oclusión microvascular, la presión hidrostática facilita la saculación de los capilares, lo cual se observa clínicamente como un microaneurisma ${ }^{8}$.

El microaneurisma es la primera lesión de la RD que puede identificarse clínicamente; su detección depende de que midan $60 \mu \mathrm{m}$ o más, para poder observarse con el oftalmoscopio. La fotografía digital actualmente permite obtener imágenes con resolución de 21 megapíxeles o mayor, con lo cual pueden identificarse microaneurismas más pequeños; sin embargo, la ausencia de microaneurismas no excluye el daño capilar.

La angiografía con fluoresceína se ha usado durante mucho tiempo para la evaluación y el manejo de la RD; consiste en la inyección de un medio de contraste (fluoresceína sódica) que permite definir la red capilar retiniana. Con este estudio pueden detectarse sitios de fuga capilar correspondientes a microaneurismas menores a $60 \mu \mathrm{m}$, no detectables con la oftalmoscopia'; a esta entidad se la conoce como RD subclínica (Fig. 1). No obstante, la angiografía con fluoresceína es un estudio invasivo que puede inducir eventos adversos de magnitud leve a grave, por lo cual no se recomienda para detectar daño retiniano preclínico en la diabetes ${ }^{10}$.

En la angiografía con fluoresceína de pacientes con $\mathrm{RD}$, las zonas de cierre capilar se observan como regiones donde no circula el medio de contraste en las fases tempranas ${ }^{11}$; un parámetro que cambia el pronóstico visual es el aumento del diámetro de la zona avascular 


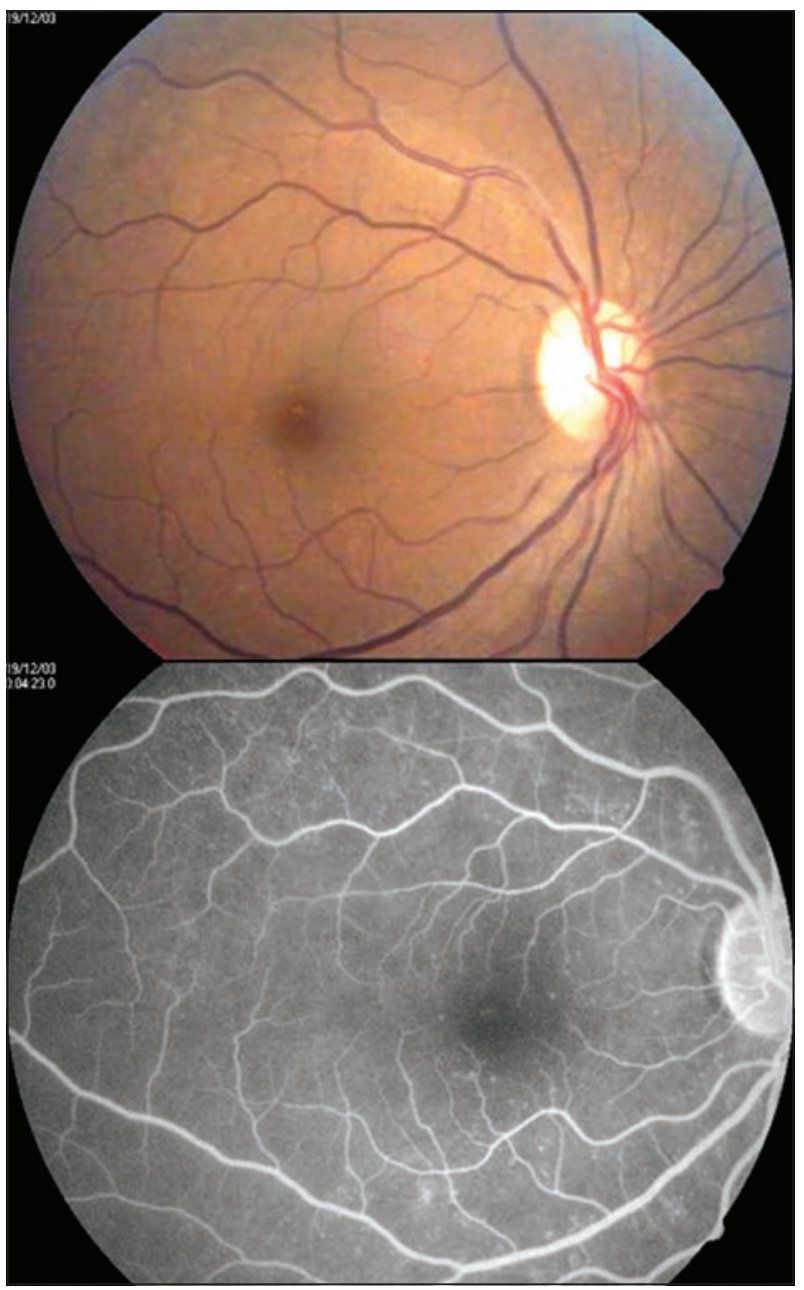

Figura 1. Retinopatía diabética subclínica. Aunque en la imagen clínica superior no se observan lesiones, la angiografía retiniana muestra zonas puntiformes hiperfluorescentes, que corresponden a microaneurismas.

foveal (ZAF), una región que en condiciones normales mide $500 \mu \mathrm{m}^{12}$. En los pacientes con diabetes sin RD la angiografía con fluoresceína no identifica cambios en la circulación del medio de contraste, por lo cual este estudio no es útil para detectar el daño microvascular previo a la formación de microaneurismas ${ }^{13}$.

\section{TOMOGRAFÍA DE COHERENCIA ÓPTICA}

Desde su introducción comercial en 1991, la TCO ha revolucionado la práctica clínica relativa al diagnóstico, tratamiento y monitoreo de las enfermedades

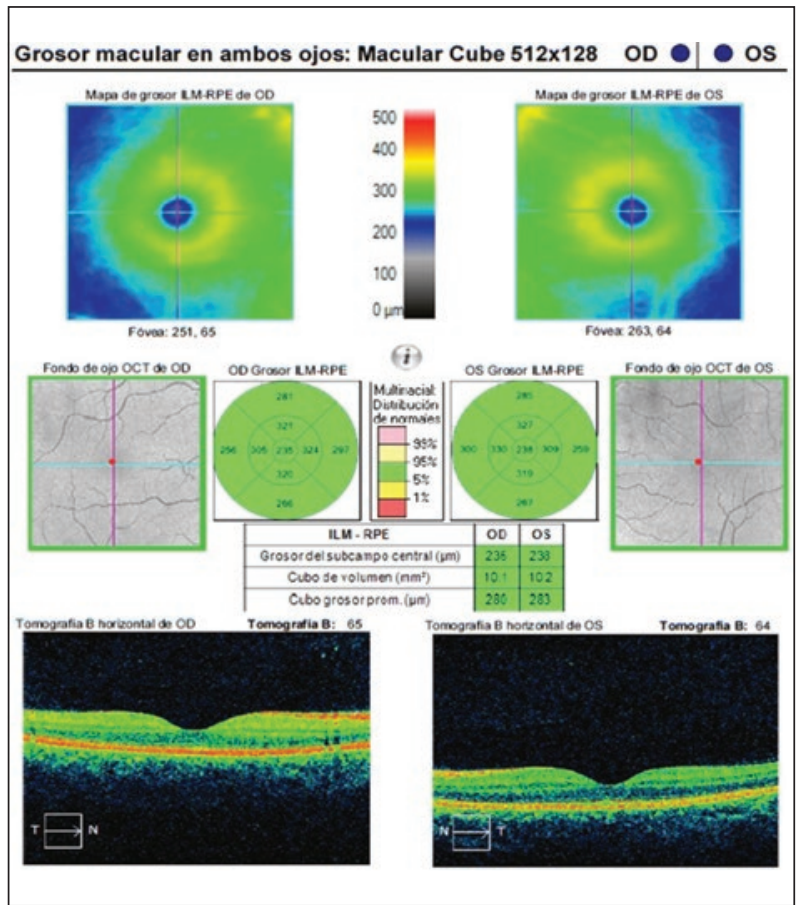

Figura 2. Mapa macular de tomografía de coherencia óptica, que mide el grosor de la retina, en tres círculos concéntricos al centro de la mácula, con diámetros de 1, 3 y $6 \mathrm{~mm}$. El mapa está dividido en campos, semejantes a los empleados por el Estudio de Tratamiento temprano de la Retinopatía Diabética para la evaluación fotográfica del fondo del ojo.

de la retina. La TCO es un estudio de imagen no invasivo que obtiene imágenes de alta resolución comparables a una biopsia óptica de la mácula, mediante la realización de cortes tomográficos micrométricos sobre el tejido, con base en el principio de interferometría ${ }^{14}$.

La TCO utiliza un interferómetro óptico que funciona agregando o interfiriendo ondas de dos rayos de luz (un diodo de infrarrojos de 820 a $830 \mathrm{~nm}$ ) de baja coherencia y calcula el tiempo de retraso y la magnitud de luz reflejada y esparcida en los tejidos, al compararlo con un haz de luz de referencia: un rayo de luz se dirige al tejido del cual se busca obtener la imagen y la señal óptica se transmite o refleja por el tejido biológico (en este caso la retina). La señal detectada por el interferómetro se procesa electrónicamente y se visualiza en una computado$\mathrm{ra}^{15}$ (Fig. 2).

Las primeras TCO empleaban un reflector en dominio de tiempo (TCO-SD), porque requerían un 
espejo móvil de referencia, por lo que solo tomaba 400 escaneos $A$ por segundo. La resolución tenía una limitación de 10 a 15 micrones, en las circunstancias más favorables, por lo que su utilidad clínica fue limitada hasta que se desarrollaron los equipos de dominio (TCO-SD), permitiendo así la visualización de 20,000-40,000 escaneos A por segundo, lo que mejoró significativamente el campo de visión, resolución de la imagen y disminución de los artefactos generados por el movimiento. Posteriormente, la TCO de fuente de barrido mejoró considerablemente la resolución, con la incorporación de una fuente de longitud de onda, no obstante, el alto costo de dicha tecnología disminuyó la aceptación comercial y clínica ${ }^{16}$.

\section{ANGIOGRAFÍA POR TOMOGRAFÍA DE COHERENCIA ÓPTICA}

La angio-TCO es una técnica reciente de imagen que permite evaluar los cambios que ocurren en los plexos capilares que rodean la mácula; utiliza el contraste del movimiento para crear imágenes de los capilares retinianos, al comparar los escaneos de descorrelación de la TCO, y genera un mapa tridimensional. Gracias a su naturaleza no invasiva y rápida se ha convertido en una herramienta de diagnóstico en la $\mathrm{RD}^{17}$.

Optovue presentó el primer equipo comercial de angio-TCO, el AngioVue, que se basó en la TCO-SD; se lanzó fuera de los EE.UU. en el 2014. Los primeros instrumentos comerciales con la tecnología Swept Source fueron introducidos por Topcon y su línea de productos Atlantis y Triton, que manejaban imágenes a 1,050 nm longitud de onda y hasta 100,000 exploraciones por segundo. Zeiss presentó el angioTCO AngioPlex, que emplea Swept-Source ${ }^{18}$.

A diferencia de la angiografía con fluoresceína, la angio-TCO no identifica zonas en las cuales falta la circulación del medio de contraste, sino las regiones donde existe circulación. Así, una región con plexos capilares permeables tiene una mayor densidad de vasos y de perfusión que otra donde no hay movimiento sanguíneo por cierre capilar.

\section{MÉTRICAS DE LA ANGIOGRAFÍA POR TOMOGRAFÍA DE COHERENCIA ÓPTICA}

El equipo Cirrus 5000 HD con AngioPlex (Zeiss, Meditec, Dublín, $(A)$, uno de los más empleados en nuestro país para el estudio de la RD, utiliza el algoritmo de la microangiografía óptica, con una velocidad de hasta 68,000 escaneos por segundo y una fuente de luz con longitud de onda centrada a $840 \mathrm{~nm}$; con ello obtiene un cubo tridimensional centrado en la mácula compuesto por 245 escaneos A. Cuenta con dos medidas de exploración automatizada del plexo capilar superficial: de $3 \times 3$ y $6 \times 6 \mathrm{~mm}$ para la densidad de vasos y de perfusión en el plexo capilar superficial, y un cubo macular estructural de 512 x 128 rastreos; los modelos más recientes pueden generar mapas de $8 \times 8$ y $12 \times 12 \mathrm{~mm}$.

La densidad de vasos y la densidad de perfusión en el mapa de $3 \times 3 \mathrm{~mm}$ se miden en tres regiones: área central ( $1 \mathrm{~mm}$ concéntrico al centro de la mácula), interna (la región entre el área central y un área de $3 \mathrm{~mm}$ concéntrica al centro de la mácula [parafóvea)] y completa (que incluye toda el área en $3 \mathrm{~mm}$ concéntricos al centro de la fóvea) (Fig. 3); la densidad interna puede dividirse en cuadrantes: superior, inferior, temporal y nasal. También pueden medirse el área, el perímetro y la circularidad de la ZAF ${ }^{19}$.

En el mapa de $6 \times 6 \mathrm{~mm}$ se mide una región comprendida hasta $6 \mathrm{~mm}$ concéntricos al centro macular (densidad externa). Su medición es más detallada entre los 3 y $6 \mathrm{~mm}$, a diferencia del mapa de $3 \times 3 \mathrm{~mm}$, que enfatiza la medición en los $3 \mathrm{~mm}$ centrales ${ }^{19}$.

\section{ZONA AVASCULAR FOVEAL}

La angio-TCO mide tres parámetros de la ZAF: área (en $\mathrm{mm}^{2}$ ), perímetro (en $\mathrm{mm}$ ) y circularidad (en proporción de 1); este último corresponde a la proporción en que la forma de la ZAF se aproxima a un círculo. La angio-TCO, al obtener imágenes de los capilares retinianos con alta resolución, permite estudiar la ZAF con mayor detalle que la angiografía con fluoresceína ${ }^{20}$. 


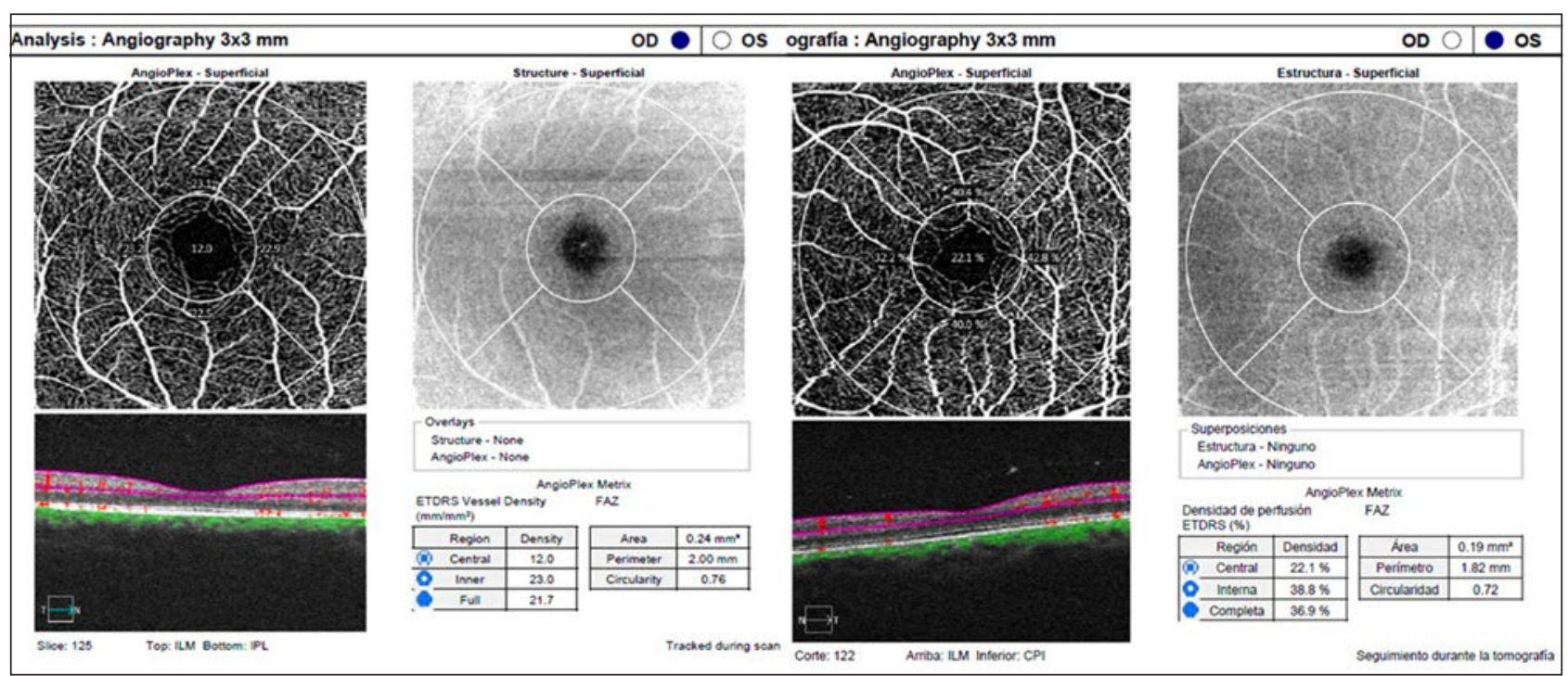

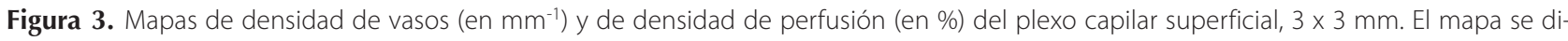
vide igual que el mapa de grosor retiniano; los cuatro campos alrededor del centro corresponden a la región parafoveal, cuya medición se reporta en el estudio como densidad interna.

Los primeros estudios reportaban que en los pacientes con diabetes sin retinopatía, el área de la ZAF era mayor que en los sujetos sin diabetes. Oliverio, et al. encontraron que el área de la ZAF aumenta significativamente en pacientes con diabetes tipo 1 , pero no en pacientes con diabetes tipo $2^{20}$. Bontzos, et al. no encontraron diferencias en el área de la ZAF entre sujetos sin diabetes y pacientes con diabetes $\sin R^{21}$.

Aunque algunos estudios han utilizado como variable de control la $Z A F$, ya que se había considerado que cuando sus características eran normales podrían excluir el daño microvascular preclínico, en pacientes mexicanos Somilleda, et al. reportaron que la circularidad de la ZAF no difiere entre sujetos con y sin diabetes tipo 2 , con y sin retinopatía, y solo tiene una baja correlación con la densidad de vasos parafoveal en pacientes con RD. Por ello, esperar a que aumente el área de la ZAF retrasa la detección tempana de microangiopatía 22 .

\section{DENSIDAD DE VASOS}

Otra variable que mide la angio-TCO es la densidad de vasos, que suma la longitud de los capilares permeables en la región evaluada, en $\mathrm{mm} / \mathrm{mm}^{2}\left(\mathrm{~mm}^{-1}\right)$; no incluye las zonas con circulación que corresponden a los vasos mayores a los capilares, otros sistemas denominan a esta variable «longitud de vasos». Durbin ${ }^{23}$, $\mathrm{Kim}^{24}$ y Choi ${ }^{25}$ encontraron que esta variable disminuye en etapas tempranas de la diabetes, antes de que se desarrolle RD, aunque no aumente la ZAF ni disminuya la agudeza visual (Tabla 1). En una muestra mexicana, Lima, et al. reportaron que la densidad de vasos parafoveal en el plexo capilar superficial en sujetos con diabetes tipo 2 sin evidencia clínica de RD era menor que en sujetos sin diabetes, aun cuando el grosor retiniano y el área de la ZAF eran normales ${ }^{26}$ (Fig. 4).

Barraso, et al. han propuesto como punto de corte de normalidad en pacientes con diabetes a una densidad de vasos de $19.15 \mathrm{~mm}^{-1}$, medida con el equipo Cirrus con AngioPlex ${ }^{27}$.

\section{DENSIDAD DE PERFUSIÓN}

Esta variable cuantifica el área de la mácula que tiene circulación, en porcentaje; a diferencia de la densidad de vasos, el porcentaje de la superficie medida en la cual existe flujo incluye el que corresponde a vasos mayores a los capilares. Otros equipos denominan a esta variable «densidad de vasos». 
Tabla 1. Comparación de variables de angiografía por tomografía de coherencia óptica entre sujetos sin diabetes y pacientes con diabetes sin retinopatía

\begin{tabular}{|c|c|c|c|c|c|}
\hline \multirow[t]{2}{*}{ Referencia } & \multicolumn{4}{|c|}{ Densidad de vasos parafoveal } & \multirow[t]{2}{*}{ Equipo } \\
\hline & Sin diabetes & $\begin{array}{l}\text { Diabetes sin } \\
\text { retinopatía }\end{array}$ & $\mathrm{p}$ & Unidad & \\
\hline $\begin{array}{l}\text { Durbin, } \\
\text { et al., } 2017^{23}\end{array}$ & $\begin{array}{c}\mathrm{n}=25 \\
\text { (mediana: 22.5; RIC: } \\
\text { 22.2-22.9) }\end{array}$ & $\begin{array}{c}n=26 \\
\text { (mediana: } 21.2 ; \text { RIC: } \\
\text { 20.5-21.9) }\end{array}$ & $<0.001^{*}$ & $\mathrm{~mm}^{-1}$ & $\begin{array}{l}\text { Cirrus } 5000 \mathrm{HD} \\
\text { AngioPlex } \\
(840 \mathrm{~nm})\end{array}$ \\
\hline Kim, et al., $2018^{24}$ & $\begin{array}{c}n=75 \\
(19.6 \pm 1.39)\end{array}$ & $\begin{array}{c}n=80 \\
(17.8 \pm 1.80)\end{array}$ & $<0.001^{+}$ & $\mathrm{mm}^{-1}$ & $\begin{array}{c}\text { Cirrus } 5000 \mathrm{HD} \\
\text { AngioPlex } \\
(840 \mathrm{~nm})\end{array}$ \\
\hline Choi, et al., $2020^{25}$ & $\begin{array}{c}n=23 \\
(21.46 \pm 3.08)\end{array}$ & $\begin{array}{c}n=28 \text { ojos } \\
(19.46 \pm 3.03)\end{array}$ & $0.014^{+}$ & $\mathrm{mm}^{-1}$ & $\begin{array}{l}\text { Cirrus } 5000 \mathrm{HD} \\
\text { AngioPlex } \\
(840 \mathrm{~nm})\end{array}$ \\
\hline
\end{tabular}

*Comparación de medias con t de Student para grupos independientes.

†U de Mann-Whitney.

RIC: rango intercuartílico.

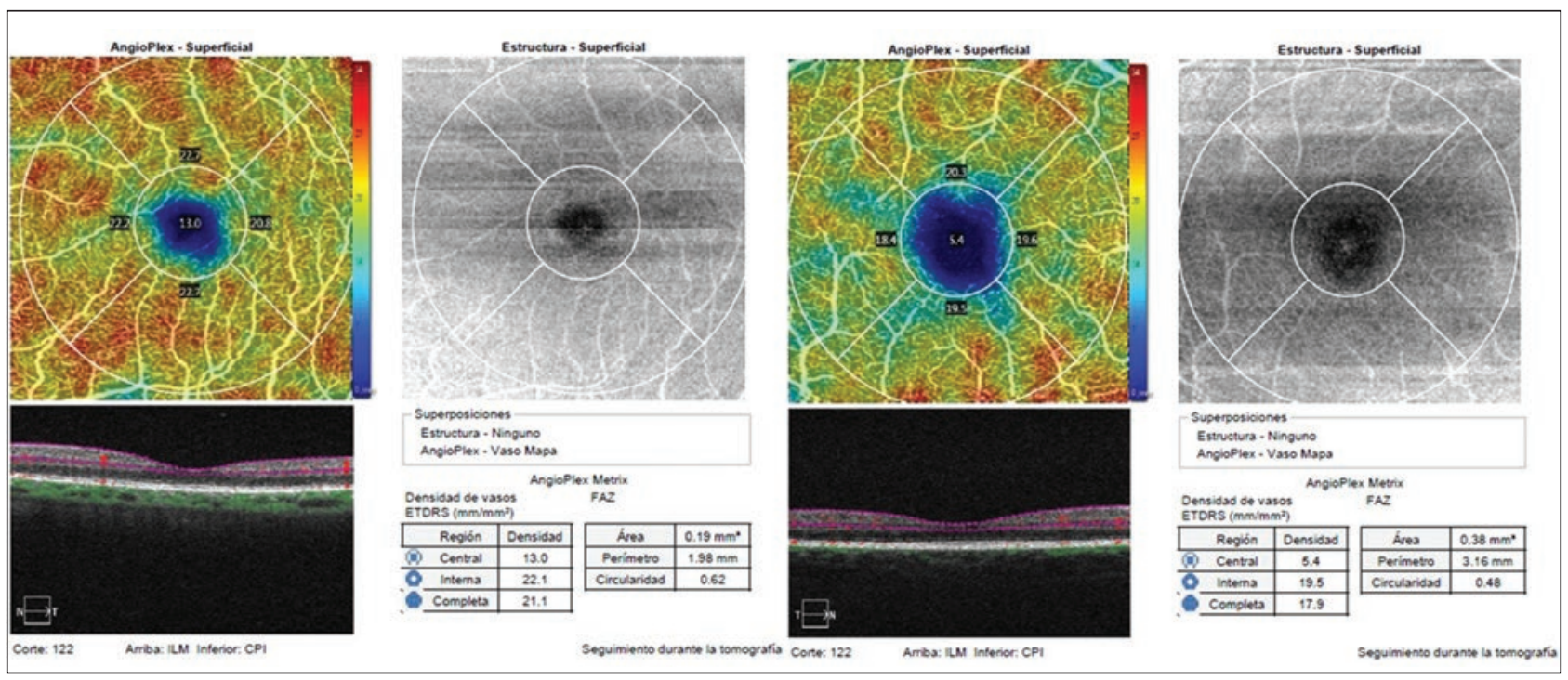

Figura 4. Mapa de densidad de vasos del plexo capilar superficial, $3 \times 3 \mathrm{~mm}$, en un sujeto sin diabetes (izquierda) y en un paciente con diabetes, sin retinopatía (derecha).

En sujetos sin diabetes de una muestra mexicana, la densidad de perfusión en el área central fue $18.7 \%$ (rango intercuartílico [RIC]: 13.9-22.5\%) y en la región interna $40.5 \%$ (RIC: $37.0 \%-41.6 \%)$, en un mapa de $3 \times 3 \mathrm{~mm}$; en pacientes con diabetes tipo 2 sin retinopatía, la densidad de perfusión central fue $14.8 \%$ (RIC: $11.8-19.9 \%$ ) y la interna $37.8 \%$ $(35.9-39.3 \%)^{28}$ (Fig. 5). Estos resultados concuerdan con los de Yang, et al. en un estudio chino, quienes también encontraron una reducción de la densidad de perfusión en la región parafoveal, pero no en la central ${ }^{29}$; Park, et al., en población coreana, en cambio, solo encontraron una reducción significativa de la densidad de perfusión en la región central, al comparar pacientes con diabetes sin retinopatía y sujetos sin diabetes ${ }^{30}$.

Simonett, et al. identificaron una disminución en la densidad de vasos parafoveal en el plexo capilar profundo en pacientes con diabetes tipo 1 comparados con controles sanos, con el mismo equipo ${ }^{31}$. Kim, et al. también reportaron una disminución en la densidad de perfusión en los plexos capilares superficiales 


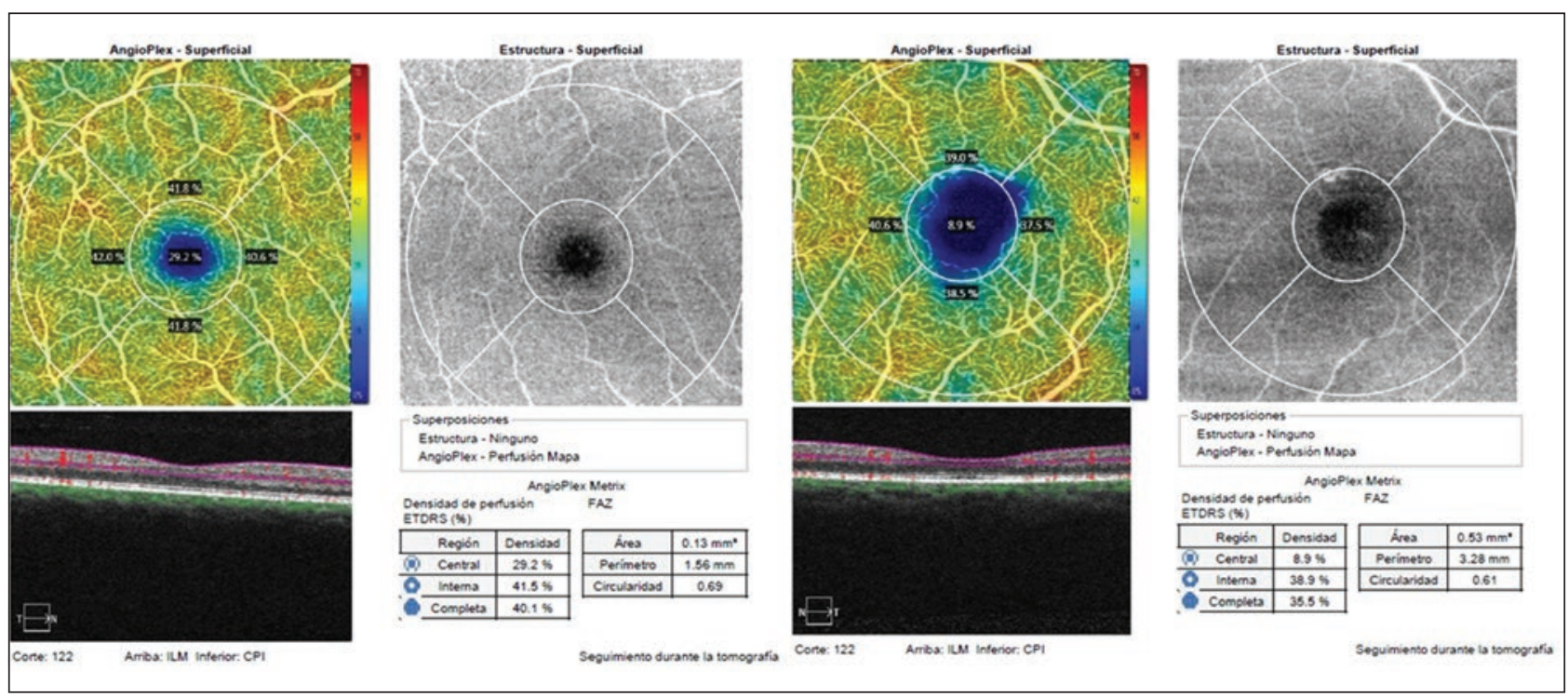

Figura 5. Mapa de densidad de perfusión del plexo capilar superficial, $3 \times 3 \mathrm{~mm}$, en un sujeto sin diabetes (izquierda) y en un paciente con diabetes, sin retinopatía (derecha).

y profundos en pacientes diabéticos sin retinopatía, con respecto a la encontrada en sujetos sanos ${ }^{24}$.

El metaanálisis de Zhang identificó que la densidad de perfusión es menor en pacientes con diabetes sin retinopatía que en los sujetos sin diabetes, en la región parafoveal, tanto en el plexo capilar superficial como en el profundo ${ }^{32}$. El punto de corte propuesto por Barraso, et al. para la densidad de perfusión normal en sujetos con diabetes es el $37 \% 27$.

En sujetos sin diabetes de otra muestra mexicana la correlación entre la densidad de vasos y la densidad de perfusión en un mapa de $3 \times 3 \mathrm{~mm}$ fue 0.98 en la región central, 0.88 en la región interna y 0.9 en la región completa (Rho de Spearman) ${ }^{33}$. Mediante el cálculo del coeficiente de determinación entre las densidades de vasos y de perfusión puede identificarse la proporción en que los capilares contribuyen al área con circulación; en la misma muestra referida, el coeficiente de determinación entre ambas variables en la región central fue $\mathrm{R}^{2}: 0.98$. Esto indica que los cambios de la densidad capilar explican el $98 \%$ de los cambios en el área con circulación, el $2 \%$ restante corresponde a cambios en la circulación de vasos mayores que los capilares; con este análisis pueden evaluarse cambios en vasos de tamaño distinto, ante variables como la edad, la altitud, iluminación e intervenciones farmacológicas.

\section{VENTAJAS Y LIMITACIONES DE LA ANGIOGRAFÍA POR TOMOGRAFÍA DE COHERENCIA ÓPTICA}

La principal fortaleza de la angio-TCO es que permite adquirir imágenes de alta resolución rápidamente (en segundos) y sin necesidad de usar medio contraste, lo cual elimina los eventos adversos por el uso de este y la convierte en un estudio seguro; otra potencial ventaja es que permite la separación y el análisis de las capas vasculares superficiales y profundas de la retina, así como la detección de cambios microvasculares $^{34}$. Además, puede capturar imágenes del flujo sanguíneo retiniano y coroideo en segundos, en comparación con la angiografía con fluoresceína, que generalmente requiere más de diez minutos; otra ventaja es que retiene los detalles distintivos de los capilares más finos sin alterarse por la variabilidad producida por el tránsito y fuga de la fluoresceína ${ }^{35}$.

Obtener una angio-TCO requiere mantener una fijación correcta el tiempo suficiente para obtener imágenes de alta calidad y disminuir los artefactos. Por otra parte, solo es capaz de producir imágenes de alta resolución del polo posterior ${ }^{36}$, aunque la evaluación de los plexos parafoveales es suficiente para detectar daño microvascular temprano. 


\section{APLICACIONES DE LA ANGIOGRAFÍA POR TOMOGRAFÍA DE COHERENCIA ÓPTICA RETINIANA EN LA EVALUACIÓN DE DAÑO MICROVASCULAR EN OTROS PLEXOS CAPILARES}

La carga invariable de la RD en la salud pública mundial destaca la importancia de continuar con la búsqueda de nuevos enfoques más allá de los estándares actuales de atención a los pacientes con diabetes ${ }^{37}$. Actualmente la densidad de vasos parafoveal es uno de los biomarcadores propuestos para el diagnóstico, pronóstico y monitorización de la RD.

Además de su utilidad para estandarizar el daño retiniano, la angio-TCO aporta información que se relaciona con la actividad de la diabetes. Entre las variables clínicas relacionadas con el daño capilar retiniano asociado con la diabetes, antes de que aparezca la retinopatía, mediante un modelo de regresión múltiple Tang, et al. identificaron al índice de masa corporal, cuyo aumento en una desviación estándar alteró la dimensión fractal de los capilares de la retina (coeficiente beta: 0.099; intervalo de confianza del 95\%; 0.011-0.187; $p=0.028)^{38}$. Choi, et al. identificaron que los niveles bajos de insulina predecían el aumento de la ZAF en los plexos capilares superficial y profundo ${ }^{25}$, y Wysocka-Mincewicz, et al. encontraron que los niveles más altos de hemoglobina glicada se asociaban con una disminución de la densidad de vasos completa ${ }^{39}$; en ambos estudios los pacientes evaluados no presentaban RD.

Zhou, et al. identificaron mediante un análisis multivariante de regresión que la glucosa plasmática en ayuno era un predictor de la densidad del plexo capilar profundo de la retina (coeficiente beta: -0.134 ; $p=0.008$ ), lo cual sugería que esta variable se encontraba correlacionada en forma independiente con la microvasculatura retiniana en sujetos $\sin R D^{40}$.

En relación con el daño en otros órganos, Kasumovic, et al. reportaron una mayor ZAF en pacientes con enfermedad renal crónica grado 4 que en pacientes con enfermedad renal crónica grado $2^{41}$. Farrah, en su revisión, refiere que la probabilidad de que una reducción en la densidad de vasos retiniana prediga la coexistencia y gravedad de la enfermedad renal crónica en diabetes, amerita un estudio más detallado ${ }^{42}$, y Zhuang, et al. reportaron una correlación de 0.304 entre la disminución de la tasa de filtración glomerular estimada y la disminución de la densidad de vasos en el plexo vascular superficial de la mácula ${ }^{43}$; el estudio de cohortes HEROIC (Heparin Requirement in Counterpulsation) sobre factores que inciden sobre la enfermedad renal diabética incluye dentro de sus variables a la angio-TCO ${ }^{44}$.

Wang, et al. reportaron que las densidades de vasos y de perfusión altas en la región macular y parafoveal se asociaban con una menor proporción urinaria albúmina/creatinina, después de hacer el ajuste por edad y longitud ocular axial; también identificaron mediante un análisis multivariante logístico multinomial que por cada reducción de $1 \mathrm{~mm}^{-1}$ en la densidad de vasos, y por cada punto porcentual de reducción de densidad de perfusión, la prevalencia de macroalbuminuria aumentaba el 11 y el 12\%, respectivamente ${ }^{45}$.

Sun, et al. reportaron que los factores de riesgo cardiometabólico se asocian con las alteraciones retinianas, y que con el equipo Cirrus con AngioPlex son más evidentes en un mapa de $3 \times 3 \mathrm{~mm}^{46}$. Zhou, et al. evaluaron variables sistémicas inductoras de estrés en sujetos sin diabetes, y plantearon que, como los vasos retinianos comparten cambios patológicos con los vasos coronarios, las condiciones de la microvasculatura retiniana son de gran valor para representar el estado de la vasculatura coronaria o sistémica, y que determinar cómo contribuyen los factores de riesgo para desarrollar enfermedad cardiovascular ateroesclerótica al desarrollo de las alteraciones capilares de la retina, es un elemento esencial para las intervenciones de prevención ${ }^{40}$.

Con respecto a otros vasos, Drinkwater, et al. reportaron que en pacientes con diabetes tipo 2 la reducción de densidad de vasos parafoveal en el plexo capilar profundo se asocia con la presencia de cualquier estenosis carotídea ipsilateral ${ }^{47}$; y Wang, et al. reportaron que la densidad de vasos del plexo capilar superficial se asocia con escalas de hiperintensidad de la materia blanca, así como con la función cognitiva de los pacientes con enfermedad de pequeños vasos cerebrales ${ }^{48}$. 


\section{CONCLUSIÓN}

La angio-TCO es una herramienta de imagen reciente que permite detectar daño microvascular retiniano cuantitativamente, antes de que se desarrolle RD. La medición de la densidad de vasos parafoveal es más recomendable que una imagen normal del fondo del ojo para descartar microangiopatía retiniana, y más segura y eficaz que la angiografía retiniana con fluoresceína, con la ventaja de que no requiere medio de contraste.

Aunque el estándar de oro para descartar RD sigue siendo la fotografía del fondo del ojo, este recurso no excluye al daño microvascular previo a que se desarrollen los hallazgos clínicos. Además de identificar cambios en la retina, la angio-TCO es un recurso no invasivo que cuantifica daño capilar, lo cual agrega valor particularmente en estudios que evalúan afección multiorgánica temprana causada por diabetes. Para optimizar la atención del paciente que vive con diabetes, la angio-TCO es una mejor herramienta que el estándar de oro, ya que su calificación cuantitativa facilita comparar el daño capilar temprano de la retina con el de otros plexos capilares.

\section{FINANCIAMIENTO}

La presente investigación no ha recibido ninguna beca específica de agencias de los sectores públicos, comercial o con ánimo de lucro.

\section{CONFLICTO DE INTERESES}

Los autores declaran no tener conflicto de intereses.

\section{RESPONSABILIDADES ÉTICAS}

Protección de personas y animales. Los autores declaran que para esta investigación no se han realizado experimentos en seres humanos ni en animales.
Confidencialidad de los datos. Los autores declaran que han seguido los protocolos de su centro de trabajo sobre la publicación de datos de pacientes.

Derecho a la privacidad y consentimiento informado. Los autores han obtenido el consentimiento informado de los pacientes y/o sujetos referidos en el artículo. Este documento obra en poder del autor de correspondencia.

\section{BIBLIOGRAFÍA}

1. Wong TY, Sabanayagam C. Strategies to tackle the global burden of diabetic retinopathy: from epidemiology to artificial intelligence. Ophthalmologica. 2020;243:9-20.

2. Graue-Hernandez EO, Rivera-de-la-Parra D, Hernandez-Jimenez S, Aguilar-Salinas CA, Kershenobich-Stalnikowitz D, Jimenez-Corona A. Prevalence and associated risk factors of diabetic retinopathy and macular oedema in patients recently diagnosed with type 2 diabetes. BMJ Open Ophthalmol. 2020;10(5):e000304.

3. Ting DS, Cheung GC, Wong TY. Diabetic retinopathy: global prevalence, major risk factors, screening practices and public health challenges: a review. Clin Exp Ophthalmol. 2016;44:260-77.

4. Flaxel CJ, Adelman RA, Bailey ST, Fawzi A, Lim Jl, Vemulakonda GA, et al. Diabetic Retinopathy Preferred Practice Pattern ${ }^{\oplus}$. Ophthalmology. 2020;127:66-145.

5. Gildea D. The diagnostic value of optical coherence tomography angiography in diabetic retinopathy: a systematic review. Int Ophthalmol. 2018;39:2413-33.

6. Batista M, Borrlli E, Sacconi R, Bandello F, Querques G. Optical coherence tomography angiography in diabetes: a review. Eur J Ophthalmol. 2020;30:411-6.

7. Roy S, Kim D. Retinal capillary basement membrane thickening: Role in the pathogenesis of diabetic retinopathy. Progr Retin Eye Res. 2020 Sep 17:100903. doi: 10.1016/j.preteyeres.2020.100903. Online ahead of print

8. Wong T, Cheung C, Larsen M, Sharma S, Simo R. Diabetic retinopathy. Nat Rev Dis Primers. 2016;2:3-17.

9. Ashraf M, Sampani K, Abdel Al O, Fleming A, Cavallerano J, Souka A, et al. Disparity of microaneurism count between ultrawide field colour imaging and ultrawidefield fluorescein angiography in eyes with diabetic retinopathy. Br J Ophthalmol. 2020;104:1762-7.

10. Khadamy J, Abri Aghdam K, Falavarjani KG. An update on optical coherence tomography angiography in diabetic retinopathy. J Ophthalmic Vis Res. 2018;13:487-97.

11. Rasta SH, Nikfarjam S, Javadzadeh A. Detection of retinal capillary nonperfusion in fundus fluorescein angiogram of diabetic retinopathy. Bioimpacts. 2015;5:183-90.

12. Sim DA, Keane PA, Zarranz-Ventura J, Fung S, Powner MB, Platteau E, et al. The effects of macular ischemia on visual acuity in diabetic retinopathy. Invest Ophthalmol Vis Sci. 2013;54:2353-60.

13. Sun Z, Yang D, Tang Z, Ng DS, Cheung CY. Optical coherence tomography angiography in diabetic retinopathy: an updated review. Eye (Lond) 2021;35:149-61.

14. Akil H, Karst S, Heisler M, Etminan M, Navajas E, Maberley D. Application of optical coherence tomography angiography in diabetic retinopathy: a comprehensive review. Can J Ophthalmol. 2019;54:519-28.

15. Spaide RF, Fujimoto JG, Waheed NK, Sadda SR, Staurenghi G. Optica coherence tomography angiography. Progr Retin Eye Res. 2018;64:1-55.

16. Kashani AH, Chen CL, Gahm JK, Zheng F, Richter GM, Rosenfeld PJ, et al. Optical coherence tomography angiography: A comprehensive review of current methods and clinical applications. Prog Retin Eye Res. 2017;60:66-100.

17. Wylęgała A, Teper S, Dobrowolski D, Wylęgała E. Optical coherence angiography: A review. Medicine. 2016;95:e4907.

18. Munk MR, Giannakaki-Zimmermann H, Berger L, Huf W, Ebneter A, Wolf $\mathrm{S}$, et al. OCT-angiography: A qualitative and quantitative comparison of 4 OCT-A devices. PLoS One. 2017;12:e0177059. 
19. Rosenfeld P, Durbin M, Roisman L, Zheng F, Miller A, Robbins G, et al. ZEISS Angioplex ${ }^{\text {TM }}$ spectral domain optical coherence tomography angiography: technical aspects. Dev Ophthalmol. 2016;56:18-29.

20. Oliverio GW, Ceravolo I, Bhatti A, Trombetta CJ. Foveal avascular zone analysis by optical coherence tomography angiography in patients with type 1 and 2 diabetes and without clinical signs of diabetic retinopathy. Int Ophthalmol. 2021;41(2):649-58.

21. Bontzos G, Kabanarou SA, Gkizis I, Ragkousis A, Xirou T, Peto T. Retinal neurodegeneration, macular circulation and morphology of the foveal avascular zone in diabetic patients: quantitative cross-sectional study using OCT-A. Acta Ophthalmol. 2021 Jan 10. doi: 10.1111/aos.14754. Online ahead of print.

22. Somilleda-Ventura SA, Razo-Blanco Hernández DM, Reyes-Calderón JA, Ceballos-Reyes GM, Lima-Gómez V. Circularity of the foveal avascular zone and its correlation with parafoveal vessel density, in subjects with and without diabetes. Cir Cir. 2019;87:390-5.

23. Durbin MK, An L, Shemonski ND, Soares M, Santos T, Lopes M, et al. Quantification of retinal microvascular density in optical coherence tomographic angiography images in diabetic retinopathy. JAMA Ophthalmol. 2017;135:370-6.

24. Kim K, Kim ES, Yu SY. Optical coherence tomography angiography analysis of foveal microvascular changes and inner retinal layer thinning in patients with diabetes. Br J Ophthalmol. 2018;102:1226-31.

25. Choi EY, Park SE, Lee SC, Koh HJ, Kim SS, Byeon AH, et al. Association between clinical biomarkers and optical coherence tomography angiography parameters in type 2 diabetes mellitus. Invest Ophthalmol Vis Sci. 2020;61:1-6.

26. Lima-Gómez V, Razo-Blanco Hernández DM, Somilleda-Ventura SA. Comparación de la densidad capilar parafoveal entre sujetos sanos y diabéticos con y sin retinopatía. Gac Med Mex. 2018;154:S30-S35.

27. Barraso M, Alé-Chilet A, Hernández T, Oliva C, Vinagre I, Ortega E, et al. Optical coherence tomography angiography in type 1 diabetes mellitus. Report 1: Diabetic Retinopathy. Transl Vis Sci Technol. 2020;9:34.

28. Serrato-Martin G, Razo Blanco Hernández DM, Somilleda-Ventura SA, Lima-Gómez V. Correlación entre la densidad de perfusión y el grosor de la capa de células ganglionares, en sujetos sin diabetes y pacientes diabéticos sin retinopatía. Rev Mex Oftalmol. 2021;95(3):95-100.

29. Yang JY, Wang Q, Yan YN, Zhou WJ, Wang YX, Wu SL, et al. Microvascular retinal changes in pre-clinical diabetic retinopathy as detected by optical coherence tomographic angiography. Graefes Arch Clin Exp Ophthalmol. 2020;258:513-20.

30. Park YG, Kim M, Roh YJ. Evaluation of foveal and parafoveal microvascular changes using optical coherence tomography angiography in type 2 diabetes patients without clinical diabetic retinopathy in South Korea. J Diabetes Res. 2020;2020:6210865.

31. Simonett JM, Scarinci F, Picconi F, Giorno P, De Geronimo D, Di Renzo A, et al. Early microvascular retinal changes in optical coherence tomography angiography in patients with type 1 diabetes mellitus. Acta Ophthalmol. 2017:95:e751-e755.

32. Zhang B, Chou Y, Zhao X, Yang J, Chen Y. Early detection of microvascular impairments with optical coherence tomography angiography in diabetic patients without clinical retinopathy: a meta-analysis. Am J Ophthalmol. 2021:222:226-37.

33. Monares-Zepeda G, Montano M, Bonilla LA, Chew-Bonilla A, Lima-Gómez V. Comparison of macular vessel and perfusion densities correla- tions between two optical coherence tomography angiography protocols. What prevents interchanging? Gac Med Mex. 2021; en prensa.

34. Tey KY, Teo K, Tan ACS, Devarajan K, Tan B, Tan J, et al. Optical coherence tomography angiography in diabetic retinopathy: a review of current applications. Eye Vis. 2019;18:6-37.

35. Khadamy J, Abri Aghdam K, Falavarjani KG. An update on optical coherence tomography angiography in diabetic retinopathy. J Ophthalmic Vis Res. 2018;13:487-97.

36. Lee J, Rosen R. Optical coherence tomography angiography in diabetes. Curr Diab Rep. 2016;16:112-4

37. Jenkins AJ, Joglekar MV, Hardikar AA, Keech AC, O'Neal DN, Januszewski AS. Biomarkers in diabetic retinopathy. Rev Diabet Stud. 2015;12: 159-95.

38. Tang FY, Chan EO, Sun Z, Wong R, Lok J, Szeto S, et al. Clinically relevant factors associated with quantitative optical coherence tomography angiography metrics in deep capillary plexus in patients with diabetes. Eye Vis Lond. 2020;7:7.

39. Wysocka-Mincewicz M, Baszyńska-Wilk M, Gołebiewska J, Olechowski A Byczyńska A, Hautz W, et al. Influence of metabolic parameters and treatment method on OCT angiography results in children with type 1 diabetes. J Diab Res. 2020;2020:4742952.

40. Zhou W, Yang J, Wang Q, Wang Y, Yan Y, Wu S, et al. Systemic stressors and retinal microvascular alterations in people without diabetes: the Kailuan eye study. Invest Ophthalmol Vis Sci. 2021;62:20.

41. Kasumovic A, Matoc I, Rebic D, Avdagic N, Halimic T. Assessment of retinal microangiopathy in chronic kidney disease patients. Med Arch. 2020;74:191-4.

42. Farrah TE, Dhillon B, Keane PA, Webb DJ, Dhaun N. The eye, the kidney, and cardiovascular disease: old concepts, better tools, and new horizons. Kidney International. 2020;98:323-42.

43. Zhuang X, Cao D, Zeng Y, Yang D, Yao J, Kuang J, et al. Associations between retinal microvasculature/microstructure and renal function in type 2 diabetes patients with early chronic kidney disease. Diab Res Clin Pract. 2020;168:IO8373.

44. McCafferty K, Caplin B, Knight S, Hockings $P$, Wheeler D, Fan SL, et al HEROIC; a 5-year observational cohort study aimed at identifying novel factors that drive diabetic kidney disease: rationale and study protocol. BMJ Open. 2020;10(9):e033923.

45. Wang Q, Liu L, Jonas JB, Gao B, Wu SL, Chen SH, et al. Albuminuria and retinal vessel density in diabetes without diabetic retinopathy: the Kailuan eye study. Acta Ophthalmol. 2020 Dec 23. doi: 10.1111/ aos.14670. Online ahead of print.

46. Sun MT, Huang S, Chan W, Craig JE, Knight LSW, Sanders P, et al. Impact of cardiometabolic factors on retinal vasculature: a $3 \times 3,6 \times 6$ and $8 \times$ $8 \mathrm{~mm}$ ocular coherence tomography angiography study. Clin Exp Ophthalmol. 2021:49(3):260-9.

47. Drinkwater JJ, Chen FK, Brooks AM, Davis BT, Turner AW, Davis TME, et al. Carotid disease and retinal optical coherence tomography parameters in type 2 diabetes: the Freemantle Diabetes Study Phase II. Diab Care. 2020;43:3034-41.

48. Wang X, Wei Q, Wu X, Cao S, Chen C, Zhang J, et al. The vessel density of the superficial retinal capillary plexus as a new biomarker in cerebra small vessel disease: an optical coherence tomography angiography study. Neurol Sci. 2021 Jan 11. doi: 10.1007/s10072-021-05038-z. Online ahead of print 